\title{
GAMMA-RAY EMITTING TEST SOURCES FOR PORTAL MONITORS USED FOR HOMELAND SECURITY
}

\author{
L. Lucas, L. Pibida*, M. Unterweger and L. Karam \\ NIST, 100 Bureau Dr. Stop 8462, Gaithersburg, MD 20899-8462
}

Received September 8 2004, amended October 5 2004, accepted October 262004

The National Institute of Standards and Technology developed a new set of test sources for testing of portal monitors against the ANSI standard N42.35-2004. The design and source specifications are provided in this work.

\section{INTRODUCTION}

With the development of the new ANSI N42.35 standard, 'Evaluation and Performance of Radiation Detection Portal Monitors for Use in Homeland Security'(1), as well as the related Test and Evaluation Protocol for Homeland Security ${ }^{(2)}$, the National Institute of Standards and Technology (NIST) has developed a set of test sources for the testing of portal monitors. The standard ${ }^{(1)}$ specifies that the test sources should be point sources (relative to the size of the portal monitors), they should be single radionuclides and encapsulated in a $0.25 \mathrm{~mm}$ thick stainless steel disc. The radionuclides required for portal monitor testing are: ${ }^{57} \mathrm{Co},{ }^{60} \mathrm{Co},{ }^{133} \mathrm{Ba}$, ${ }^{137} \mathrm{Cs},{ }^{228} \mathrm{Th}$ and ${ }^{241} \mathrm{Am}$. These sources are useable not only for testing and evaluating instrumentation, but can also be used as calibration test sources and for instrument user training.

\section{SOURCE SPECIFICATIONS}

The sources specified in the ANSI N42.35 standard were chosen according to the half-life and gammaray energies of the radionuclide. Since radioactive sources decay over time, the use of a radionuclide with a long half-life, $\mathrm{T}_{1 / 2}$, is required if a source is to be useable for an extended period of time. For the types of detectors to be used in homeland security applications, gamma-ray energies from $50 \mathrm{keV}$ to $3 \mathrm{MeV}$ are desired. In addition, the source design requires specific encapsulation and geometry considerations. The durability of the source and resistance to shocks, extreme temperatures and rough handling must be considered; durability is a crucial aspect of the source design adopted by NIST.

The ANSI standard for testing portal monitors specifies both the construction of the source and its nominal activity at the time of testing. The actual activity in each source at the time of the testing may

*Corresponding author: leticia.pibida@nist.gov be between 0.8 and 1.2 times (i.e. $\pm 20 \%$ ) the nominal activity. The specified activities are given in Table 1.

The radioactive material, with the activity listed in Table 1, is welded between two $0.25 \mathrm{~mm}$ thick 316 stainless steel discs. Stainless steel was chosen for its chemical resistance and mechanical strength, so that a source could tolerate the force of a vehicle driven over it without loss of radioactivity. Note that the measured gamma-ray emission rate is reduced due to the attenuation in the stainless steel disc.

The source itself consists of the radioactive material, originally as a calibrated solution dissolved in acid, weighed out and dried on a piece of glass-fibre filter paper, $1.27 \mathrm{~cm}$ in diameter and $1 \mathrm{~mm}$ thick. The filter paper is placed between two 316 stainless steel discs, each $0.25 \mathrm{~mm}$ thick and $38.1 \mathrm{~mm}$ in diameter (see Figure 1 for a diagram of the discs). Before welding the stainless steel discs together, the distribution of the radioactive material in the source is verified using photo-stimulable luminescence

Table 1. Radionuclides and activities for the portal monitor test sources.

\begin{tabular}{lccc}
\hline Radionuclide & \multicolumn{3}{c}{ Activity at the time of testing (MBq) } \\
\cline { 2 - 4 } & Minimum & Nominal & Maximum \\
\hline $\begin{array}{l}{ }^{57} \mathrm{Co} \\
\left(T_{1 / 2}=270 \mathrm{~d}\right)\end{array}$ & 2.75 & 3.44 & 4.13 \\
${ }^{60} \mathrm{Co}$ & & & \\
$\left(T_{1 / 2}=5.2714 \mathrm{y}\right)$ & 0.119 & 0.148 & 0.177 \\
${ }^{133} \mathrm{Ba}$ & 0.681 & 0.851 & 1.021 \\
$\left(T_{1 / 2}=10.51 \mathrm{y}\right)$ & & & \\
${ }^{137} \mathrm{Cs}$ & 0.474 & 0.592 & 0.710 \\
$\left(T_{1 / 2}=30.07 \mathrm{y}\right)$ & & & \\
${ }^{228} \mathrm{Th}$ & 0.208 & 0.259 & 0.310 \\
$\left(T_{1 / 2}=1.9116 \mathrm{y}\right)$ & & & \\
${ }^{241} \mathrm{Am}$ & 13.7 & 17.1 & 20.5 \\
$\left(T_{1 / 2}=432.2 \mathrm{y}\right)$ & & & \\
\hline
\end{tabular}




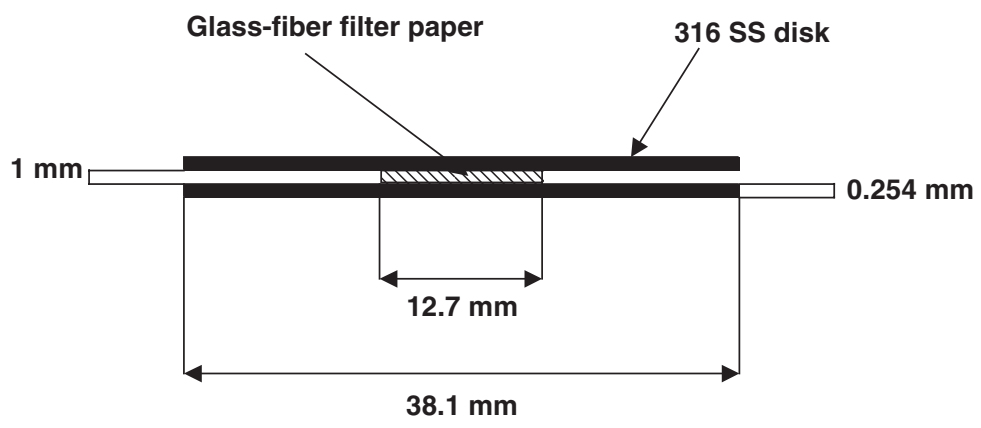

Figure 1. Test source before welding.

imaging techniques [Fuji system], and the activity is measured using high purity germanium (HPGe) detectors. The measured activity is compared to the activity calculated from the mass of the original NIST calibrated radioactive solution. Then the discs are welded around the edge using an automated tungsten-inert-gas (TIG) welding system. Each welded source is subsequently measured again using the HPGe detectors to verify that the welding process did not alter the activity. The stainless steel disc is then mounted in a 6061-T6 aluminium holder. The holder consists of two plates, each $5.08 \mathrm{~cm}$ wide, $8.89 \mathrm{~cm}$ long and $0.3175 \mathrm{~cm}$ thick with a $3.175 \mathrm{~cm}$ hole for mounting the source, and three smaller holes for mounting the holder to the experimental set up for testing the portal monitor. See Figure 2 for a diagram of the holder. Part of the holder area is used for labeling the source, including source number, activity and reference date. The first set of sources produced by NIST is pictured in Figure 3. The technical drawings for the sources are given in Appendix 1.

\section{FUTURE CONSIDERATIONS}

For the commercialisation and widespread distribution of this type of test source, a variety of additional issues must be addressed. The National Institute of Standards and Technology has developed the prototype, and the first set of test sources, for the testing and verification of portal monitors with minimal concern as to the cost and availability of the radioactive materials. If mass production is desired in future, the cost of the radioactive material to be used, as well as its availability in large quantities, will play a major role in the practicality of specific radionuclides to be used. In addition, the role that manufacturers will play in the production and distribution of these sources must be considered. We are aware of five companies that currently produce or distribute radioactive sources in the USA: AEA

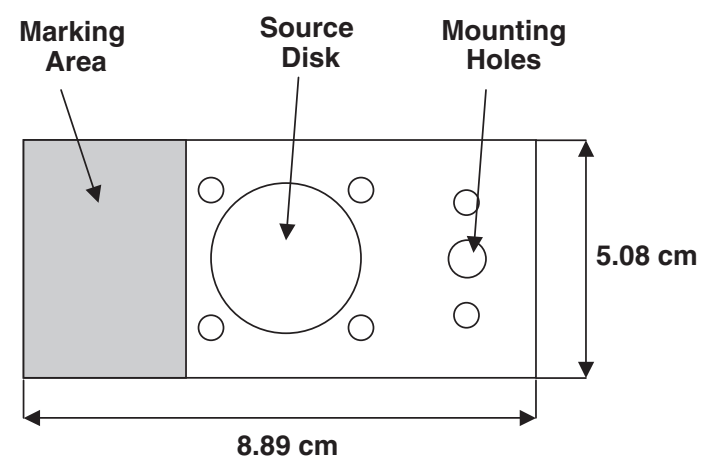

Figure 2. Aluminium holder.

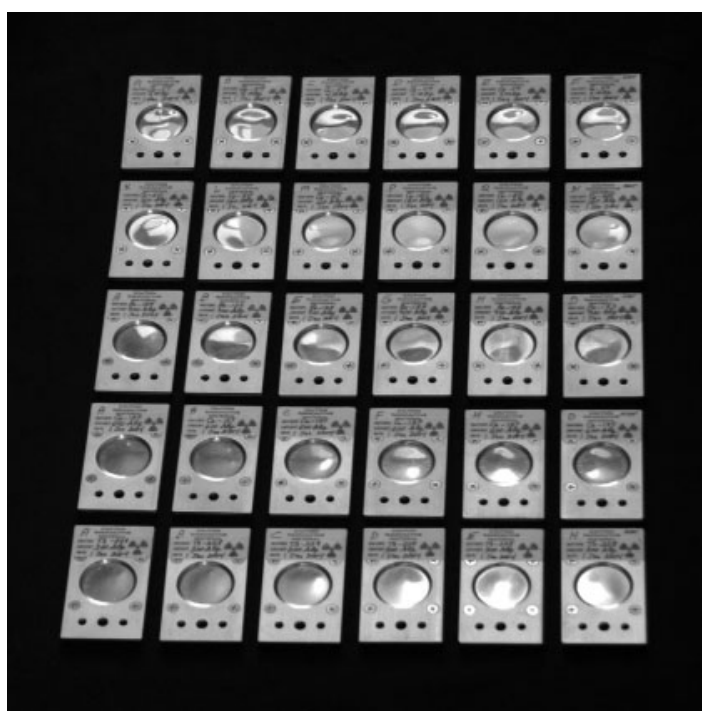

Figure 3. Picture of the first set of test sources. 


\section{LUCAS ET AL.}

Technologies, Analytics, Isotope Products Laboratories, North American Scientific and Spectrum Techniques, Inc. Several of these companies are participants in a Measurement Assurance Programme (MAP) with NIST to assure traceability to the US national standards in the production of sources for nuclear power plant and medical applications. A similar and more extended programme could be implemented for homeland security applications. These and other issues will need to be resolved before there can be an effective programme to distribute these types of sources more widely.

\section{CONCLUSIONS}

The first set of test check sources have been produced and sent to the Oak Ridge National Lab (ORNL), the Pacific Northwest National Lab (PNNL), the Los Alamos National Lab (LANL), the Lawrence Livermore National Lab (LLNL) and the Environmental Measurement Lab (EML), where they are being used for the testing and evaluation of commercially available portal monitors. There is still the need to discuss with source manufacturers the requirements and logistics for larger scale production, if needed.

\section{REFERENCES}

1. ANSI N42.35-2004, American National Standard Evaluation and Performance of Radiation Detection Portal Monitors for Use in Homeland Security (2004).

2. T\&E Protocol N42.35, April 2004, Testing and Evaluation Protocol of Radiation Detection Portal Monitors for Use in Homeland Security. DHS T\&E N42.35 (2004).

\section{APPENDIX 1. TECHNICAL DRAWINGS FOR THE SOURCES}

Figures A.1-A.4 and Table A.1 show in detail the technical drawings for the sources.

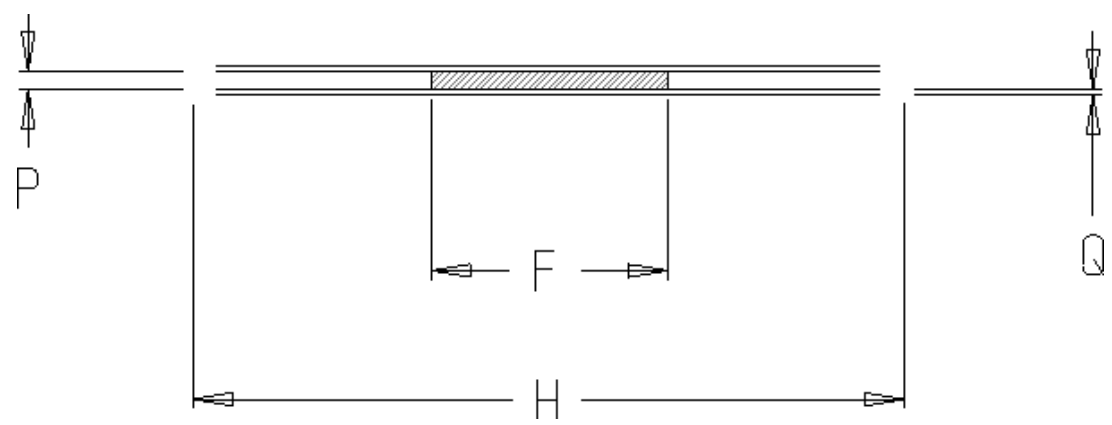

Figure A.1. DHS test source disc before welding.

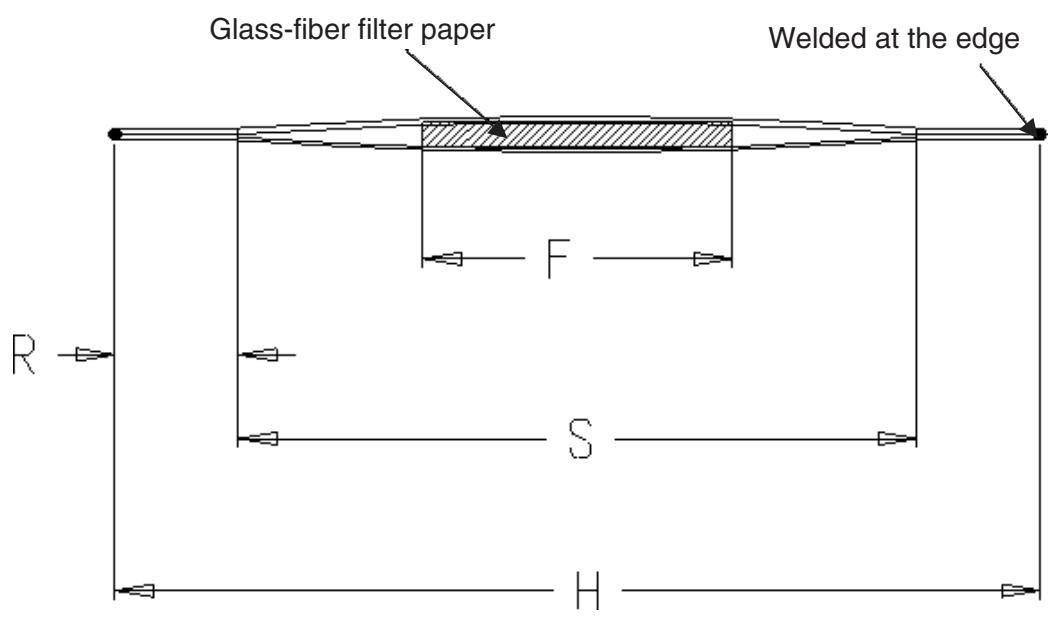

Figure A.2. DHS test source disc after welding. 

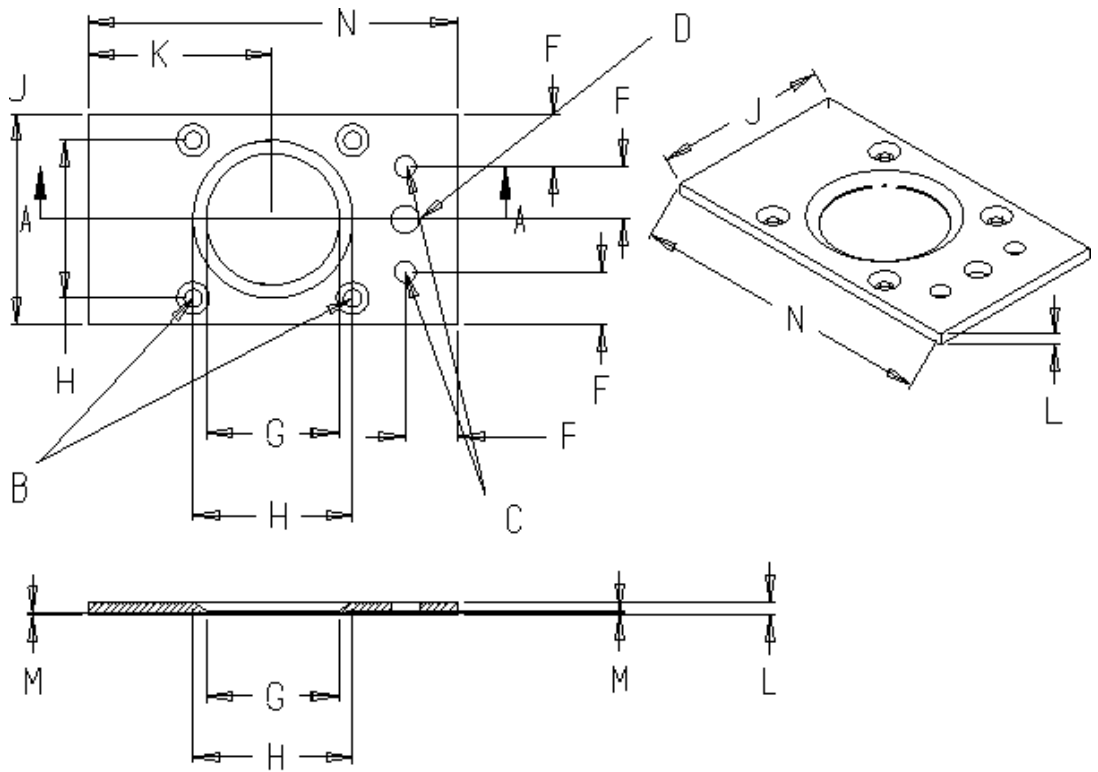

SECTION A-A

Figure A.3. Top plate for radioactive DHS test source holder.

Table A.1. List of drawing dimensions.

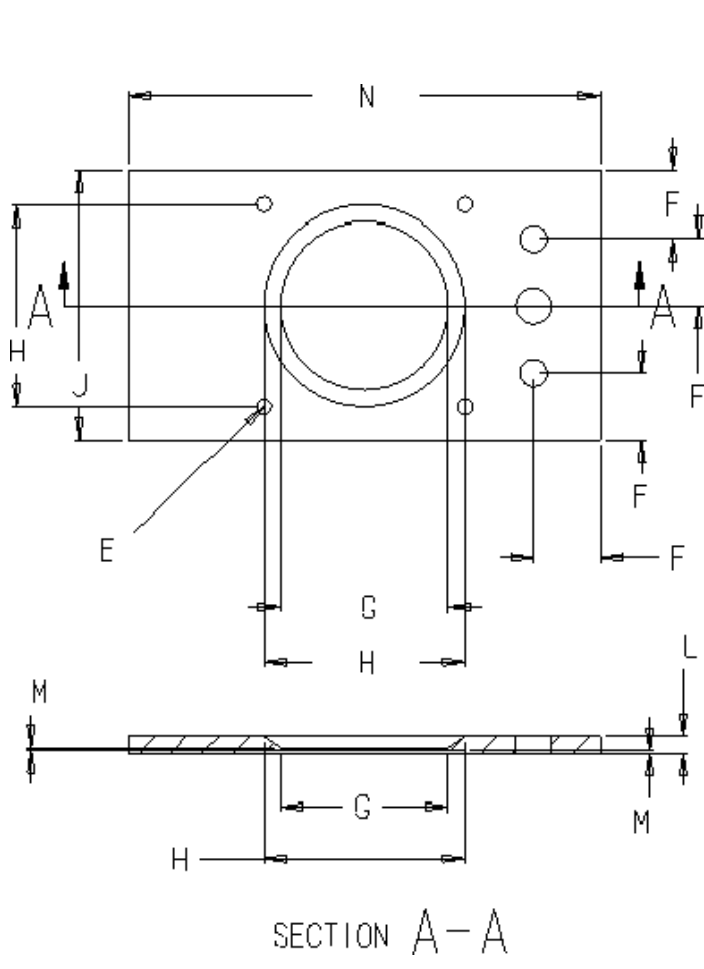

Figure A.4. Bottom plate for radioactive DHS test source holder.

\begin{tabular}{ll}
\hline Dimension & \multicolumn{1}{c}{ Values in millimeters } \\
\hline B & $\begin{array}{l}\text { Drill through } 3.6576 \mathrm{~mm} \text { diameter } \\
\text { and counter-sink for \#6-32 flat head } \\
\text { machine screws (4 places) }\end{array}$ \\
C & $\begin{array}{l}\text { Drill through 5.1562 mm diameter } \\
\text { (2 places) }\end{array}$ \\
D & Drill through 6.7564 mm diameter \\
& (1 place) \\
E & Drill through and tapped for \#6-32 \\
& machine screw (4 places) \\
F & 12.70 \\
$\mathrm{G}$ & 31.75 \\
$\mathrm{H}$ & 38.10 \\
$\mathrm{~J}$ & 50.80 \\
$\mathrm{~K}$ & 44.45 \\
$\mathrm{~L}$ & 3.175 \\
$\mathrm{M}$ & 0.508 \\
$\mathrm{~N}$ & 88.90 \\
$\mathrm{P}$ & 1.00 \\
$\mathrm{Q}$ & 0.254 \\
$\mathrm{R}$ & 5.08 \\
$\mathrm{~S}$ & 27.94 \\
\hline
\end{tabular}

Note: Material test source disc $=316$ stainless steel.

Material test source holder $=6061$-T6 Aluminium bar 3.175 $\times 50.8 \times 88.9 \mathrm{~mm}$.

Tolerance $= \pm 0.254 \mathrm{~mm}$ (unless otherwise indicated).

Tolerance for the counter-bore depth dimension " $\mathrm{M}$ " of $0.508 \mathrm{~mm}$ is $\pm 0.127 \mathrm{~mm}$.

Tolerance for stainless steel disc thickness is $\pm 0.0127 \mathrm{~mm}$ (0.0005 inches). 